\title{
A reliable method for bonding polydimethylsiloxane (PDMS) to polymethylmethacrylate (PMMA) and its application in micropumps
}

\author{
Hsih Yin Tan ${ }^{\mathrm{a}}$, Weng Keong Loke ${ }^{\mathrm{a}}$, Nam-Trung Nguyen ${ }^{\mathrm{b}, *}$ \\ ${ }^{a}$ DSO National Laboratories, 20 Science Park Drive, Singapore 118230 \\ ${ }^{b}$ School of Mechanical and Aerospace Engineering, Nanyang Technological University, 50 \\ Nanyang Avenue, Singapore, 639798. Tel: (+65) 67904457, Fax: (+65) 67911859
}

\begin{abstract}
Poly(methyl methacrylate) (PMMA) attracts growing interest in microfluidics research community due to its low cost, high transparency, good mechanical and chemical properties. The more flexible polydimethylsiloxane (PDMS) is well suited for pneumatic actuation. However, PDMS is permeable to gases and absorbs molecules from the sample liquids. Combining PMMA with PDMS would allow a microfluidic device to utilize advantages of both materials. Bonding PMMA to PDMS is a critical step for this hybrid approach. In this paper, we present a simple, fast and reliable technique for bonding PMMA to PDMS. A 25- $\mu$ m thick adhesive film (ARclearß Optically clear adhesive 8154, Adhesive Research, Glen Rock, PA USA) was laminated onto a clean PMMA surface. Subsequently, pre-cured PDMS mixture was spin coated onto the adhesive film. After curing, the adhesive and
\end{abstract}

\footnotetext{
*Corresponding author

Email addresses: thsihyin@dso.org.sg (Hsih Yin Tan), 1wengkeo@dso.org.sg (Weng Keong Loke), mntnguyen@ntu.edu.sg (Nam-Trung Nguyen)

URL: http://www.ntu.edu.sg/home/mntnguyen (Nam-Trung Nguyen)
} 
the PDMS layer form a hybrid membrane. The bonding quality and the strength of the PDMS/adhesive membrane was tested using a precision pressure source. A peristaltic micropump was fabricated by bonding a PDMS part with microchannels to the PDMS/PMMA part. The PDMS/adhesive membrane acts as the pneumatic actuator for the micropump. Pressurised air was switched to the three pneumatic actuators by solenoid valves and control electronics. The micropumps can achieve a flow rate as high as $96 \mu \mathrm{l} / \mathrm{min}$. The techniques reported in this paper allow the integration of microfluidic components made of both PMMA and PDMS in a single device.

Keywords: microfluidics, polymethylmethacrylate (PMMA), polydimethylsiloxane (PDMS), bonding, micropump, pneumatic actuation 


\section{Introduction}

Since its emergence in the 1980s, microfluidics has been widely used in the development of systems such as microreactors for chemical, biological processes and lab-on-a-chip (LOC) for chemical and biochemical analysis $[1,2]$. Valving, concentrating and separation of molecules in a minute amount of liquid and on a single device are challenging tasks in this development. These challenges lead to the necessity of miniaturising active functions and the integration of microfluidic components such as microvalves, micropumps, microsieves and micromixers $[1,2,3]$. Silicon and glass were the first substrate materials utilised for the fabrication of microfluidic devices. Despite the advantages of using glass and silicon as substrate materials for microchips fabrication, these devices have limitations such as the high cost due to their specialised fabrication processes. Recently, the rapid expansion of microfluidics-based biochip market leads to the need of cost effective materials and fabrication techniques. The low cost also allows the single use of these devices. Polymers have become one of the favoured materials for microfluidic devices, as they possess excellent optical properties. Polymers are disposable, biocompatible, easy and cost-effective to fabricate and suitable for mass fabrication. A variety of polymeric materials with unique physical and chemical characteristics is available. Some of these materials are thermoplastic polymers such as polymethyl methacrylate (PMMA), polycarbonate (PC), cyclic olefin copolymer (COC) or cyclic olefin polymer (COP) and elastomers such as polydimethylsiloxane (PDMS). Both PMMA and PDMS are popular in the fabrication of microfluidic devices.

PDMS is widely used for both prototypes and commercial devices. Mi- 
crochannels are usually fabricated in PDMS using soft lithography approach [4]. With a low Young's modulus of approximately $750 \mathrm{kPa}$, PDMS is flexible, permeable to gas and compatible to most non-organic solvents. These properties make PDMS suitable for the fabrication of pneumatic microvalves. A flexible PDMS membrane is an important component of peristaltic micropumps. Multi-layer soft-lithography process for microfluidic systems was reported by Unger et al. [5]. In these systems, the microchannels are separated from the pneumatic chambers by a thin PDMS membrane [6]. Zhang et. al. [7] reported a multi-layer micropump consisting of a thin layer of PDMS sandwiched between two PMMA layers. The PDMS layer acts as the actuating membrane and the PMMA layers accommodate both pneumatic chambers and microchannels. The PDMS/PMMA bond was not strong and can be easily detached. Kim et. al. [8] modified PDMS with polypyrrole, a conductive polymer, to create a membrane that was encased in a PMMA device.

Besides PDMS, PMMA is another popular polymeric material for microfluidic devices. This thermoplastic is cheap, easy to mass produce, rigid, transparent, impermeable to air and compatible with electrokinetics. PMMA can be machined using hot embossing, injection moulding, laser ablation and solvent imprinting [9].

Bonding polymeric materials to obtain sealed microfluidic devices was one of the technological challenges [10]. The simplest and cheapest form of bonding two PMMA substrates is adhesive bonding. However, liquid adhesives can clog the microchannels. Partially cured PDMS as an adhesive layer for bonding PMMA was also investigated [11]. Thermal fusion bonding is a 
commonly used technique for fabricating PMMA devices $[12,13,14,15,16]$. An advantage of direct thermal bonding is the homogenous surface property when identical materials are used for both the substrate with microchannels and the cover layer. Another technique is solvent bonding. Chlorocarbon solvents such as chloromethane dichloromethane and chloroform can be used for bonding PMMA pieces. Similar to bonding with liquid adhesive, these organic solvents often clog the microchannels as they can dissolve PMMA easily. Lin et. al. [17] reported the use of low azeotropic solvent to bond PMMA microfluidic devices without clogging the microchannels.

Due to the different surface properties of PDMS and PMMA, bonding them is not an easy task. It is not possible to initiate irreversible bond by treating both surfaces with oxygen plasma. A number of technologies were developed for bonding PDMS to PMMA such as surface modification by chemical vapor deposition (CVD), silane/silicate coating and adhesive coating $[18,19,20]$. A few research works reported the modification of PMMA surface with silanization. This method gives the modified PMMA surface a silane matrix similar to glass, allowing it to be treated by oxygen plasma and bonded to PDMS. Vlachopoulou et. al. [19] reported a method of bonding PMMA to PDMS using surface treatment of PMMA with aminopropyltriethoxysilane (APTES). The bond was irreversible. However, contact with water causes the Si-O-C bond to hydrolyse. Lee et. al. treated the PMMA surface with isopropxy modified by bis-trimethoxy-silyl-propyl-amine, which is capable of forming hydrolytically stable bonds between PDMS and modified PMMA [20]. Recently, Suzuki et al. [21] spin-coated slica gel to form a glass-like surface on thermoplastics including PMMA, PC, polyvinyl chloride 
(PVC) and polypropylene (PP). The bonding strengths between PDMS and these coated thermoplastics were comparable to that between PDMS and glass.

This paper reports a novel method of bonding between PMMA and PDMS using an adhesive film. A hybrid membrane consisting of an adhesive layer and a PDMS layer works was used as the pneumatic actuator in a peristaltic micropump. The performance of our pump is compared to peristaltic pumps reported in recent years $[22,23,24,25,26]$. This bonding technique reported here is easy to implement and cost effective. Furthermore, contact to aqueous media does not cause the PMMA/adhesive/PDMS bond to delaminate.

\section{Materials and methods}

\subsection{Materials}

PMMA (optical grade, Ying Kwang Acrylic Trading, Singapore) was used as the substrate for the membrane test devices and micropumps. The PMMA material has a refractive index of 1.49 , a haze of $0.5 \%$ and light transmission of $92 \%$. The base polymer and the curing agent of PDMS was purchased from Dow Corning (Sylgard 184). The $25 \mu \mathrm{m}$ thick adhesive (ARclearß) Optically clear adhesive 8154, Adhesive Research, Glen Rock, PA USA) is a doublefaced acrylic acid-free, ultra low-outgassing bonding tape for fabrication of seals with adhesive-free zones. Peel adhesion of the adhesive is $5.45 \mathrm{~N} / \mathrm{cm}$. This adhesive is commonly used in the production of flat panel displays, large and small liquid crystal displays (LCDs) and touch screens. According to its data sheet, the adhesive is suitable for bonding of flexible to flexible or flexible to rigid optical components. The material is provided as a $25-\mu \mathrm{m}$ adhesive 
film sandwiched between a $50-\mu \mathrm{m}$ and a $37.5-\mu \mathrm{m}$ thick polyester liner. The adhesive film has a refractive index of 1.47 , a haze of $2 \%$ and a visible light transmission of $98 \%$. Thus, this film is suitable for microfluidic applications with optical detection. Pressurised nitrogen (SOXAL, Singapore) was used as the pressure source for the membrane tests. Two-way electromagnetic solenoid valves for controlling the pressure were used (LEE Co., Westbrook, CT, USA).

\subsection{Fabrication process}

Figure 1 depicts the fabrication process of a PMMA/adhesive/PDMS device. The process starts with micromachining of the PMMA substrate. The devices (test membranes and micropumps) were designed using AutoCad 2006 and transferred to a commercial $\mathrm{CO}_{2}$ laser system (Universal M-300 Laser Platform, Universal Laser system Inc. Arizona, USA). The laser engraved the pneumatic chambers and the microchannels on the PMMA substrate. The $\mathrm{CO}_{2}$ laser has a wavelength of $10.6 \mu \mathrm{m}$ and a maximum power of $25 \mathrm{~W}$. The maximum speed of the laser beam is $640 \mathrm{~mm} / \mathrm{s}$. The different channel heights can be adjusted by the corresponding laser power and scanning speed. This form of fabrication is fast and cost effective. After laser machining, the PMMA pieces are washed in $70 \%$ ethanol and rinsed with de-ionised (DI) water. Other fabrication techniques such as hot embossing or moulding can be used to form channel structures on the PMMA substrate.

Figures 1(a-c) depict the fabrication process of the devices with the hybrid adhesive/PDMS membrane. The PMMA substrate was cut into the form of a circular 4-inch wafer. The pneumatic chambers are then engraved into the PMMA wafer. After cleaning, the dry adhesive film was laminated on the 
wafer. The dry acrylic adhesive can bond well to the PMMA surface. PDMS was prepared, degassed and spin coated onto the PMMA wafer covered by the adhesive film. At a rate of $1500 \mathrm{rpm}$ and spinning time of 30 seconds, a PDMS film of $50 \mu \mathrm{m}$ was formed on the adhesive layer. Thermal curing of the adhesive/PDMS layer took place at $80{ }^{\circ} \mathrm{C}$ for one hour. For the membrane tests, the fabrication process ended here. For the fabrication of the peristaltic micropumps, the PMMA wafer with the adhesive/PDMS membrane and the PDMS part with the microchannels underwent oxygen plasma treatment, and were subsequently bonded together. The process results in an irreversible bond.

The PDMS part with microchannels was fabricated using soft lithography. Similar to the pneumatic chambers, the microfluidic patterns were designed using AutoCad, and subsequently transferred on a polymeric mask using a high-resolution laser printer. A clean 4-inch silicon wafer (Bonda, Singapore) was spin coated with the photoresist SU8-100 (Microchem, Newton, MA, USA) at $2000 \mathrm{rpm}$ to yield a thickness of $150 \mu \mathrm{m}$. The wafer subsequently underwent soft bake at $65^{\circ} \mathrm{C}$ and $95^{\circ} \mathrm{C}$ before exposure of ultra violet (UV) light at an intensity of $8.9 \mathrm{~mW} / \mathrm{cm}^{2}$ for 67 seconds using an mask aligner and exposure unit (Karl Suss, MA6/BA6). After the exposure, the wafer was baked at $65^{\circ} \mathrm{C}$ and $95^{\circ} \mathrm{C}$. The wafer was then developed for 15 minutes before rinsing with IPA (Isopropyl alcholol, Sigma) followed by hard baking at $150^{\circ} \mathrm{C}$. De-gased PDMS mixture prepared with a ratio of 10:1 was poured over the SU-8 mould and cured at $80^{\circ} \mathrm{C}$ for one hour. This cured PDMS part was treated with oxygen plasma before bonding to the PDMS/adhesive membrane as described above. 


\section{Results and Discussions}

\subsection{Characterization of adhesive/PDMS membrane}

Figure 2 shows the designs of the membranes used in the pressure test. The test chips contain different pneumatic chambers with the shape of squares and circles. The edges or the diameters are $300 \mu \mathrm{m}, 500 \mu \mathrm{m}, 1000 \mu \mathrm{m}$, $1500 \mu \mathrm{m}, 2000 \mu \mathrm{m}, 2500 \mu \mathrm{m}$ and $3000 \mu \mathrm{m}$. The depth of the pneumatic chambers in PMMA is approximately $500 \mu \mathrm{m}$. All pneumatic chambers share the same pressure port.

Investigations of the adhesive/PDMS membrane of both circular and square pneumatic chambers were carried out by using a calibrated pressure source. Pressurised nitrogen was first connected to a pressure controller (PPC4 DH Instrument, Fluke Co., USA) which works as the pressure source for the tested membranes. A $45^{\circ}$ mirror (Edmund Optics, Singapore) was placed next to the membrane array to allow imaging both top view and side view of the deformed membrane, Figure 2(c). The deformation of the membrane was captured by a CCD camera and the corresponding software (Video Savant, IO Industries, Inc., Canada). Images of the deformed membranes were captured, and then analysed by a program written in Matlab (MathWorks, MA, USA). The centre deflection and the radius of curvature of the deformed membrane was measured at different applied pressures, which vary from $2 \mathrm{kPa}$ to $20 \mathrm{kPa}$. The pressure range for the square pneumatic chambers is from $2 \mathrm{kPa}$ to $16 \mathrm{kPa}$. The pressure was first increased to the maximum value and then decreased back to the initial value, to test the possible hysteresis behavior of the hybrid membrane due to the failure of the adhesive film at high pressure. Figure 3 shows the typical images of deformed 
membranes of both circular and square cases.

Figure 4 shows the centre deflection and the radius of curvature of circular membranes with different diameters. The results show that the bond quality between PDMS and PMMA is good. No delamination occurs even at the maximum pressure of $20 \mathrm{kPa}$. At higher pressures, the membrane would burst while the bond was still intact. The results also indicate that the larger the applied pressure the higher is the centre deflection of the membrane. At the same applied pressure, a larger membrane experiences a larger deflection. The relatively large deflection would allow the adhesive/PDMS membrane to be used as a pneumatic actuator for microvalves and micropumps. The radius of curvature of the deformed membrane decreases with increasing pressure, Fig. 4(b). For membranes with diameters less than $2 \mathrm{~mm}$, the centre deflection does not show hysteresis indicating that the adhesive film is intact in the tested pressure range. For large membranes with diameters of more than $2.5 \mathrm{~mm}$, the centre deflection versus pressure curve shows a hysteresis behaviour. Beyond a critical pressure of about $14 \mathrm{kPa}$ (3-mm membrane) and $17 \mathrm{kPa}$ (2.5-mm membrane), the slope of the deflection curve changes significantly. This change and the hysteresis may be caused by the tear-off effect of the more rigid adhesive layer, making the overall membrane becomes softer. At higher pressures (beyond $16 \mathrm{kPa}$ for 3 -mm membrane and beyond $18 \mathrm{kPa}$ for 2.5-mm membrane), the slope decreases again, Fig. 4(a). In this regime, the deformed membrane take the shape of a baloon and the non-linear stiffening effect may take place.

The square membranes were tested up to $16 \mathrm{kPa}$. Similar to the circular membranes, an increasing pressure increases the centre deflection of the 
membrane and decreases its radius of curvature. Clear hysteresis behavior is observed with the 3-mm membrane. Due to the larger membrane area, the deflection for the square membrane $\left(A=a^{2}\right)$ was significantly larger than that of a circular membrane $\left(A=\pi a^{2} / 4\right)$. As shown in the inserts of Figures 4 and $5, a$ is the diameter of the circular membrane and the edge of the square membrane. At $20 \mathrm{kPa}$, a 3-mm circular membrane reaches a centre deflection of approximately $3.5 \mathrm{~mm}$. At $16 \mathrm{kPa}$, a $3-\mathrm{mm}$ square membrane reaches a centre deflection of approximately $4 \mathrm{~mm}$. Although the membrane was deformed and took the shape of a balloon, the membrane did not detach from the PMMA substrate. For the same design footprint, a square membrane would allow a larger deflection. The square membrane was therefore used for designing the peristaltic pump described in the next section. The irreversible damage to the adhesive film caused by high pressure is clearly indicated by the hysteresis behavior. For most microfluidic applications, the membrane does not need to be stretched to the shape of a balloon. Thus, the hybrid adhesive/PDMS membrane is suitable for pneumatic actuation.

The thickness of the hybrid membrane is limited by the $25-\mu \mathrm{m}$ thick adhesive layer. The thickness of the PDMS layer can be controlled by the spin speed and the spinning time. As shown in the membrane characterization above, the thick membrane needs a relatively large area to warrant large deflection at a relatively low actuation pressure. If the PDMS layer is $10 \mu \mathrm{m}$ thick, the membrane size can be reduced to $100 \mu \mathrm{m}$ to $500 \mu \mathrm{m}$.

\subsection{Operation and characterization of the peristaltic micropump}

As shown in Fig. 1(e), a peristaltic pump was fabricated by bonding the PMMA wafer with the adhesive/PDMS membrane on a PDMS part 
containing the microchannel of the pump. When the rigid PMMA pneumatic chambers are filled with compressed air, the the membrane deflects to the bottom of the microchannel, which has a height of only $150 \mu \mathrm{m}$. Fluid flow inside the microchannel is sealed off and displaced by combining the peristaltic motion of the three actuating membrane as depicted in Fig, 6. The pneumatic chambers in PMMA are placed $1 \mathrm{~mm}$ apart. The overlapping area between the pneumatic chamber and the microchannel defines the actuating membrane which is depicted as the grey areas in the schematics of Fig. 6 . The area of the actuating membrane is $A=W_{\mathrm{a}} \times W_{\mathrm{c}}$, where $W_{\mathrm{a}}$ and $W_{\mathrm{c}}$ are the widths of the pneumatic chamber and the microchannels, respectively. Two different widths of pneumatic chamber $W_{\mathrm{a}}=1 \mathrm{~mm}$ and $2 \mathrm{~mm}$ and three different channel widths of $W_{\mathrm{c}}=0.5 \mathrm{~mm}, 0.8 \mathrm{~mm}$ and $1.0 \mathrm{~mm}$ were tested. DI water mixed with red food dye was used as the test liquid. The micropumps were activated by three two-way solenoid valves. The valves are latched when the direction of the applied current is changed. A customized switching circuit using relays was built for this purpose, Fig. 6. As the circuit requirement is similar to that used by Nguyen et al. [27], a slight modification was carried out for driving the peristaltic micropump. With the two-way solenoid valve, air from a pressure source is diverted between the pneumatic chamber in PMMA and the environment. The control circuit is connected to a data acquisition (DAQ) module (National Instruments, Texas, USA). Figure 6 depicts the hardware setup for testing the micropump.

LabVIEW 8.5 with NI-DAQmx 8.8 (National Instruments, Texas, USA) were used as the platform to run the control program of the micropump. Transistor-transistor-logics (TTL) signals are sent from the DAQ module to 
Table 1: Actuating sequences of the three membranes in the peristaltic micropump.

\begin{tabular}{llll}
\hline Sequence & Membrane 1 & Membrane 2 & Membrane 3 \\
\hline 1 & 0 & 0 & 0 \\
2 & 1 & 0 & 0 \\
3 & 1 & 1 & 0 \\
4 & 1 & 1 & 1 \\
5 & 0 & 1 & 1 \\
6 & 0 & 0 & 1 \\
\hline
\end{tabular}

the relay circuit. In order to pump the liquid in one direction, the three actuating membranes are controlled in a peristaltic manner. Table 1 shows the actuating sequences of the peristaltic micropump. The "on" and "off" states are indicated as "1" and "0", respectively. The six states listed in Table 1 form one pumping cycle. Therefore, if the pump is operating at a frequency of $f$, each state should last $1 /(6 f)$ seconds.

In the characterization experiments, the pumping frequency was varied. Compressed air at $138 \mathrm{kPa}$ works as the pressure source. The average pumping velocity $v=x / t$ was determined by measuring the displacement of the front meniscus $x$ in the tubing connected to the pump and the corresponding time $t$. With the known cross-sectional area $A$ of the tubing, the flow rate can be determined as $Q=v \times A$. In the experiments, the measurement time was fixed at $t=120 \mathrm{sec}$. The uncertainties in measuring the time and the displacement are $\Delta t=0.1 \mathrm{sec}$ and $x=0.5 \mathrm{~mm}$. The error propagation for the average pumping velocity can be determined as:

$$
\Delta v=\frac{\Delta x}{t}+\frac{x}{t^{2}} \Delta t=\frac{\Delta x}{t}+\frac{v}{t} \Delta t .
$$


Thus, the error in the measured velocity consists of two components: $\Delta v=$ $\Delta x / t$ from length measurement and the relative error $\Delta v / v=\Delta t / t$ from time measurement. Applying this error analysis to our experiment, the systematic error contributed by the length measurement is $0.5 \mu \mathrm{l} / \mathrm{min}$ and the relative systematic error contributed by the time measurement is $0.8 \%$.

Figure 7(a) shows the pump characteristics with an pneumatic chamber width of $W_{\mathrm{a}}=1 \mathrm{~mm}$. The results show that at the same pumping frequency the larger the channel width, the higher is the flow rate. At frequencies beyond $20 \mathrm{~Hz}$, the flow rate remains constant or even drops slightly in the case of channel width of $1 \mathrm{~mm}$. This phenomenon is caused by the limited dynamics of the switching relays. A solid-state relays with a higher dynamics would allow a higher pumping flow rate at frequency beyond $20 \mathrm{~Hz}$. Figure 7(b) shows the performance of micropumps with a larger pneumatic chamber width of $W_{\mathrm{a}}=2 \mathrm{~mm}$. For smaller channel width of $W_{\mathrm{c}}=0.5 \mathrm{~mm}$ and $0.8 \mathrm{~mm}$, the twice as large actuating membranes almost double the flow rate as compared to the performance of pumps with $W_{\mathrm{c}}=1 \mathrm{~mm}$. For the larger microchannel with $W_{\mathrm{c}}=1 \mathrm{~mm}$, the improvement in flow rate is not that apparent.

During the test, no leakage of air from the pneumatic chamber into the microchannel was observed. This phenomenon was a serious problem in pneumatic pumps made entirely of PDMS. Furthermore, no delamination of the adhesive/PDMS membrane was observed. The adhesive/PDMS membrane was impermeable to air and capable of withstanding high air pressure. For the largest microchannel with $W_{\mathrm{a}}=1 \mathrm{~mm}$, micropumps were capable of delivering a maximum flow rate of $89 \mu \mathrm{l} / \mathrm{min}$ and $96 \mu \mathrm{l} / \mathrm{min}$ for $W_{\mathrm{a}}=1 \mathrm{~mm}$ 
and with $W_{\mathrm{a}}=2 \mathrm{~mm}$, respectively. To test the long-term stability, the pump with $W_{\mathrm{c}}=0.5 \mathrm{~mm}$ and $W_{\mathrm{a}}=2 \mathrm{~mm}$ was tested at pumping frequencies of $10 \mathrm{~Hz}$ and $30 \mathrm{~Hz}$ for 3 days each. The flow rates ware sampled during this time span and depicted in Fig. 8. The results show that the flow rate distribution is consistent with the error analysis and the data depicted in Fig. 7.

Figure 9 compares the performance of the peristaltic pump reported in this paper with its counterparts recently reported in the literature by Unger et al. [5], Ok and Satoshi [26], Yang et al. [22], Huang et al. [23], Liu et al. [24] and Lee et al. [25]. The chart plots the performance of the pump based on the maximum flow rate and the pumping frequency of this flow rate. Since the peristaltic pumping is a displacement concept, for a given pump design, the flow rate is higher with higher pumping frequency. A pump with high performance (high throughput) will be located at the top left corner of the chart. The line in the chart represents the linear fit of the coordinates of our pump and the pumps of Ok and Satoshi [26] as well as of Yang et al. [22]. Fig. 9 shows that our pump belongs to the upper performance class among peristaltic micropumps.

\section{Conclusions}

We report a novel technique of bonding PMMA to PDMS in a muti-layer polymeric microfluidic device. This technique is based on the concept of laminating a thin layer of dry adhesive on the PMMA substrate followed by spin coating a layer of pre-cured PDMS mixture. These layers then undergo thermal curing followed by oxygen plasma to bond irreversibly to another 
PDMS part. This fabrication technique is suitable for batch processes, and the thickness of the membrane is controllable. Different pneumatic chamber sizes and different air pressures were shown to affect the deflection of the membrane. For the same design foot print, square membranes were able to deflect more than the circular membranes. The square membranes were used in peristaltic micropumps as pneumatic actuators. Pumping of liquid was successful. The highest flow rate of $96 \mu \mathrm{l} / \mathrm{min}$ was achieved by the largest pneumatic chamber and channel at a pumping frequency of $10 \mathrm{~Hz}$. This novel fabrication technique of the micropumps prevents bubble formation in the microchannel from the infusion of air from the actuation chambers. The layer of adhesive is impermeable to air and adhere well to the PMMA surface. The bond between PMMA and PDMS is strong and reliable as the different layers did not delaminate in the different tests reported in this paper. The micropumps based on the combination of PMMA, dry adhesive and PDMS is powerful, and suitable to work with other components in a more complex LOC platform.

\section{Acknowledgements}

Financial support from Agency for Science, Technology and Research (A*STAR) and Defence Science and Technology Agency (DSTA) of Singapore is gratefully acknowledged (Grant number 08/1/50/19/590, "ScreenPrinted Microfluidic Platform With Pneumatic Micropumps and Microvalve for Synchronized Regeneration and Detection of Nerve Agent in Human Blood Samples"). The authors would like to thank Chin-Hong Ooi and VinhNguyen Phan for writing the LabView and Matlab programs. 


\section{References}

[1] S. Haeberle, R. Zengerle, Microfluidic platforms for lab-on-a-chip applications, Lab Chip 7 (2007) 1094-1110.

[2] C. Fredickson, Z. Fan, Macro-to-micro interfaces for microfluidic devices, Lab Chip 4 (2004) 526-533.

[3] K. Oh, C. Anh, A review of microvalves, J. Micromech. Microeng. 16 (2004) R13-R39.

[4] S. Sia, G. Whitesides, Microfluidic devices fabricated in poly(dimethylsiloxane) for biological studies, Electrophoresis 24 (2003) 3563-3576.

[5] M. Unger, H. Chou, T. Thorsen, A. Scherer, S. Quake, Monolithic microfabricated valves and pumps by multilayer soft lithography, Science 288 (2000) 113-116.

[6] T. Thorsen, S. Maerkl, S. Quake, Microfluidic large scale integration, Science 298 (2002) 580-584.

[7] W. Zhang, S. Lin, C. Wang, J. Hu, Z. Zhuang, Y. Zhou, R. Mathies, C. Yang, Pmma/pdms valves and pumps for disposable microfluidics, Lap Chip 9 (2009) 3088-3094.

[8] J. Kim, K. Lau, R. Shepherd, Y. Wu, G. Wallace, D. Diamond, Performance characteristics of a polypyrrole modified polydimethylsiloxane (pdms) membrane based microfluidi pump, Sensors and Actuators A 148 (2008) 239-244. 
[9] P. Abgrall, L. Low, N. Nguyen, Fabrication of planar nanofluidic channels in a thermoplastic by hot-embossing and thermal bonding, Lab Chip 7 (2007) 520-522.

[10] C. Tsao, D. DeVoe, Bonding of thermoplastic polymer microfluidics, Micofluid. Nanofluid. 6 (2007) 1-16.

[11] W. Chow, K. Lei, G. Shi, W. Li, Q. Huang, Microfluidic channel fabrication by pdms-interface bonding, Smart Mater. Struct. 15 (2006) S112-S116.

[12] H. Tan, W. Loke, Y. Tan, N. Nguyen, A lab on a chip for detection of nerve agent sarin in blood, Lab Chip 8 (2008) 885-891.

[13] Y. Sun, Y. Kwok, N. Nguyen, Low pressure, high temperature thermal bonding of polymeric microfluidic devices and their applications for electrophoretic separation, J. Micromech. Microeng. 16 (2006) 1681-1688.

[14] X. Zhu, G. Liu, Y. Guo, Y. Tian, Study of pmma thermal bonding, Microsyst. Tech. 13 (2007) 403-407.

[15] Z. Chen, Y. Gao, R. Su, C. Li, J. Lin, Fabrication and characterization of poly(methyl methacrylate) microchannels by in situ polymerization with a novel metal template, Electrophoresis 24 (2003) 3246-3252.

[16] Y. Chen, L. Zhang, G. Chen, Fabrication, modification, and application of poly(methyl methacrylate) microfluidic chips, Electrophoresis 29 (2008) 1801-1814. 
[17] C. Lin, C. Chao, C. Lan, Low azeotropic solvent for bonding of pmma microfluidic devices, Sensors and Actuators B 121 (2007) 698-705.

[18] A. Toh, S. Ng, Z. Wang, Fabrication and testing of embedded microvalves within pmma microfluidic devices, Microsyst. Techno. 15 (2009) 1335-1342.

[19] M. Vlachopoulou, A. Tserepi, P. Pavli, P. Argitis, M. Sanopoulou, K. Misiakos, A low temperature surface modification assisted method for bonding plastic substrates, J. Micromech. Microeng. 19 (2009) 015007.

[20] K. Lee, R. Ram, Plastic-pdms bonding for high pressure hydrolytically stable active microfluidics, Lab Chip 9 (2009) 1618-1624.

[21] Y. Suzuki, M. Yamada, M. Seki, Sol-gel based fabrication of hydbrid microfluidic devices composed of pdms and thermoplastic substrates, Sensors and Actuators B 148 (2010) 323-329.

[22] Y. Yang, S. Hsiung, G. Lee, A pneumatic micropump incorporated with a normally closed valve capable of generating a high pumping rate and a high back pressure, Microfluid Nanofluid 6 (2009) 823833.

[23] S. Huang, M. Wu, Z. Cui, G. Lee, A membrane-based serpentine-shape pneumatic micropump with pumping performance modulated by fluidic resistance, J. Micromech. Microeng. 18 (2008) 045008.

[24] C. Liu, J. Rick, T. Chou, , H. Lee, G. Lee, Integrated microfluidic system for electrochemical sensing of urinary proteins, Biomed. Microdevices 11 (2008) 201-211. 
[25] Y. Lee, K. Lien, H. Lei, G. Lee, An integrated microfluidic system for rapid diagnosis of dengue virus infection, Biosensors and Bioelectronics 25 (2009) 745-752.

[26] C. Ok, K. Satoshi, Fabrication of a peristaltic micro pump with novel cascaded actuators, J. Micromech. Microeng. 18 (2008) 025022.

[27] N. Nguyen, K. Ng, X. Huang, Manipulation of ferrofluid droplets using planar coils, Applied Physics Letter 89 (2006) 052509. 


\section{Biographies}

Hsih Yin Tan received her B. Eng. degree from Nanyang Technological University in 2005. She is employed by the DSO National Laboratory and currently working toward her M. Eng. degree.

Weng Keong Loke received his B. Sc, M. Sc and Ph. D. degrees from National University of Singapore in 1992, 1996 and 2005, respectively. He is a distinguished member of technical staff and head of medical countermeasures (chemicals and toxins) laboratory in chemical, biological and radiological defence program of DSO National Laboratory, Singapore.

Nam-Trung Nguyen received his Dip-Ing, Dr. Ing and Dr. Ing Habil degrees from Chemnitz University of Technology, Germany, in 1993, 1997, and 2004, respectively. In 1998, he worked as a postdoctoral research engineer in the Berkeley Sensor and Actuator center (UC Berkeley, USA). He is currently an Associate Professor with the School of Mechanical and Aerospace Engineering of the Nanyang Technological University in Singapore. His research is focused on microfluidics and instrumentation for biomedical applications. The second edition of his book "Fundamentals and Applications of Microfluidics" and the recent books "Micromixers" and "Nanofluidics" were published in 2006, 2008 and 2009, respectively. 


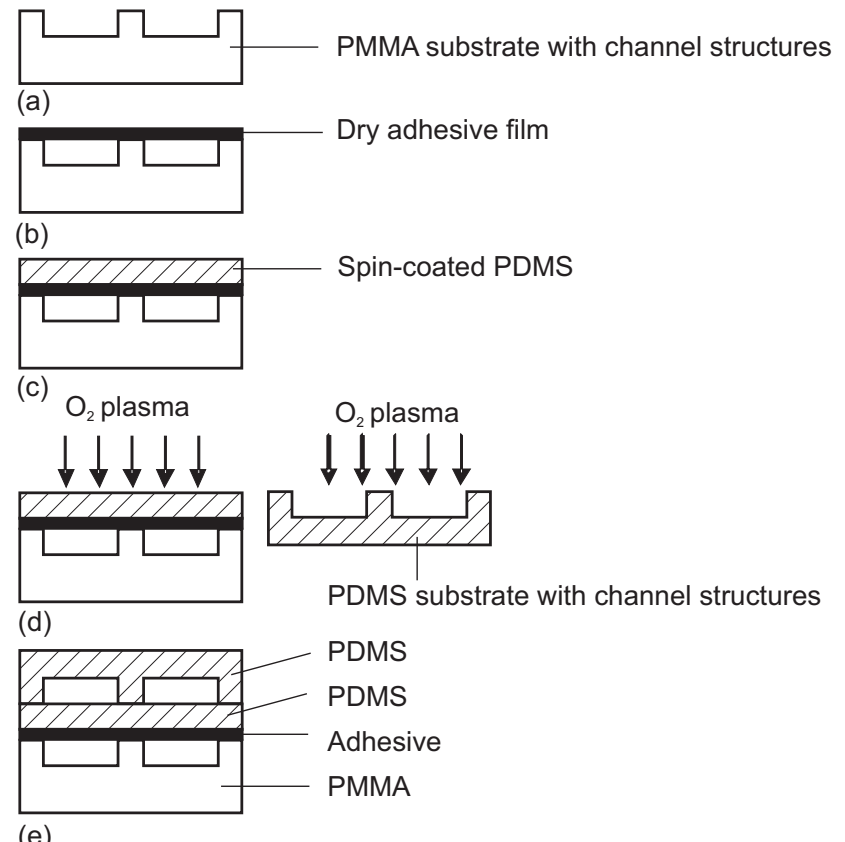

Figure 1: Fabrication process of the PMMA/adhesive/PDMS part and the peristaltic micropump: (a) PMMA wafer with engraved pneumatic chambers; (b) Dry adhesive film is laminated onto the PMMA pneumatic chambers; (c) PDMS is spin coated onto the PMMA wafer covered by a dry adhesive; (d) The PMMA wafer with the adhesive/PDMS membrane and the moulded PDMS part with microchannel are treated in oxygen plasma; (e) Treated surfaces are bonded together to form the micropump with actuation chambers in PMMA and microchannel in PDMS. 


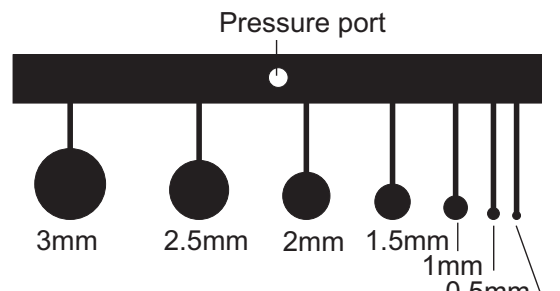

(a) $\quad$ Pressure port $\quad 0.5 \mathrm{~mm}$
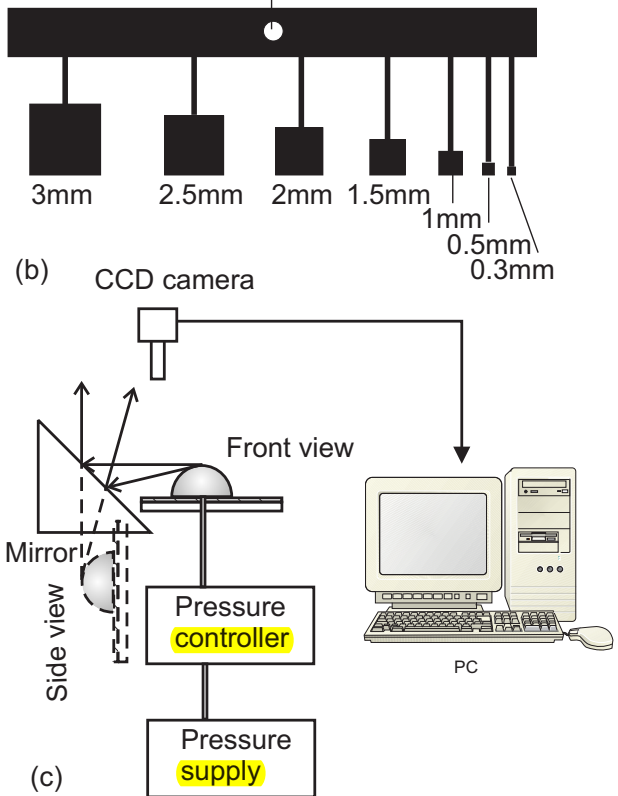

Figure 2: PDMS membrane designs for the pressure test: (a) Circular pneumatic chamber; (b) Rectangular pneumatic chamber; (c) Experimental setup. 


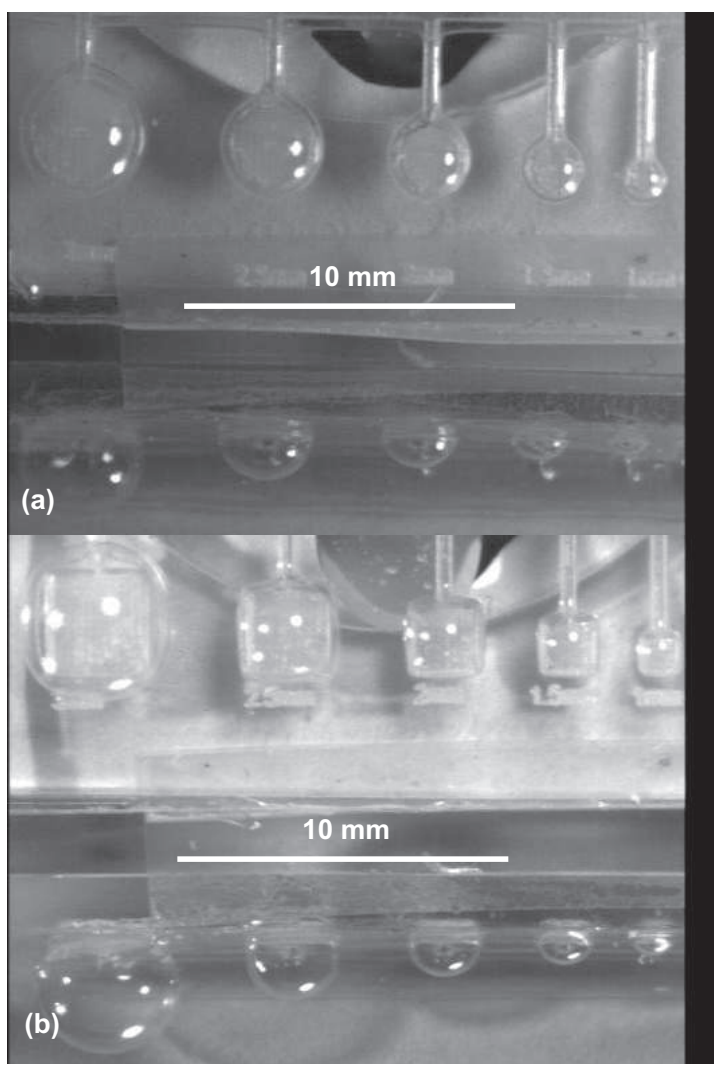

Figure 3: Deformed membranes under applied pressure: (a) Circular membranes at $20 \mathrm{kPa}$; (b) Square membrane at $15 \mathrm{kPa}$. 

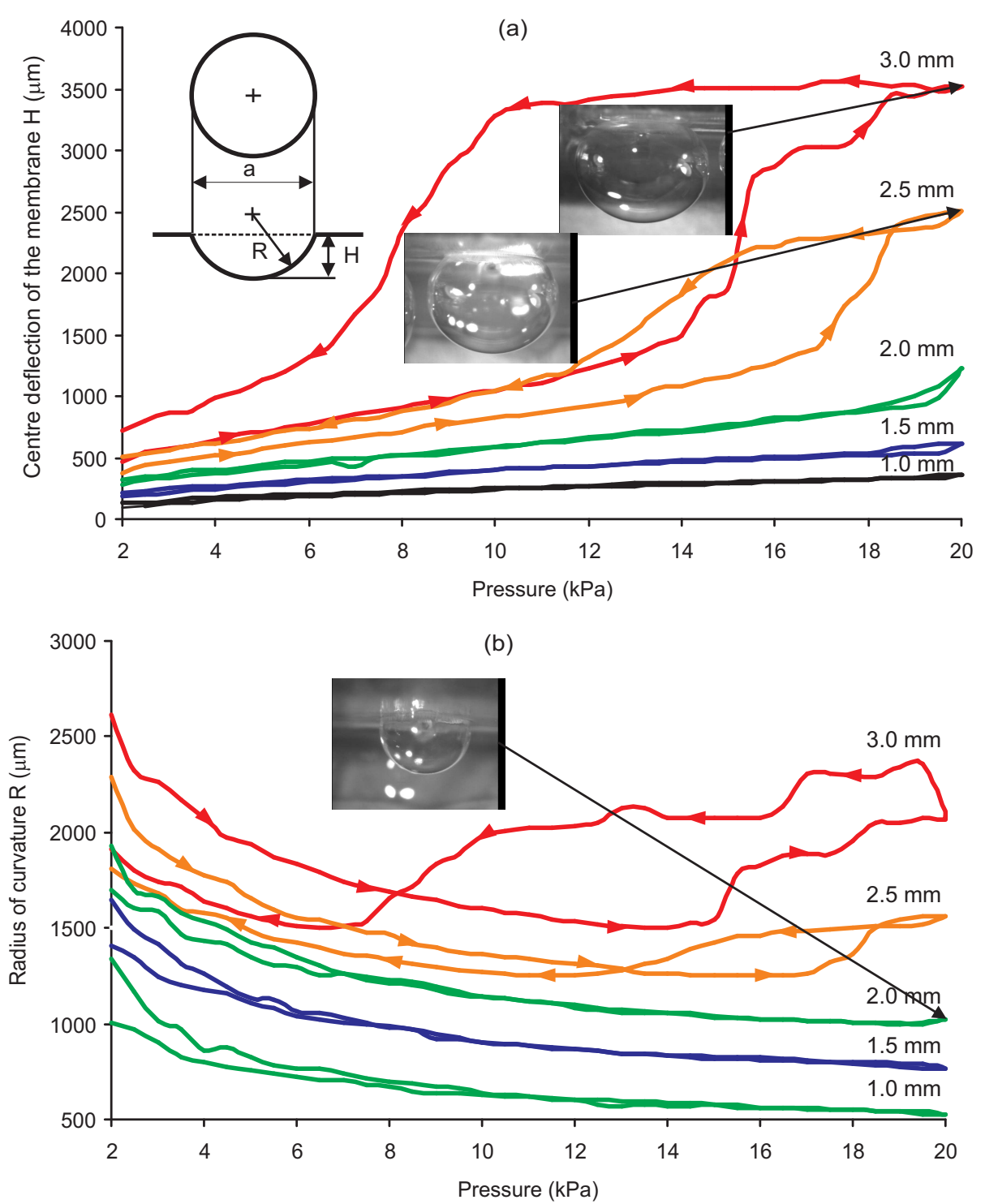

Figure 4: Geometry of circular membranes at different applied pressures (the membrane diameter is indicated next to the respective curve): (a) Centre deflection; (b) Radius of curvature. 

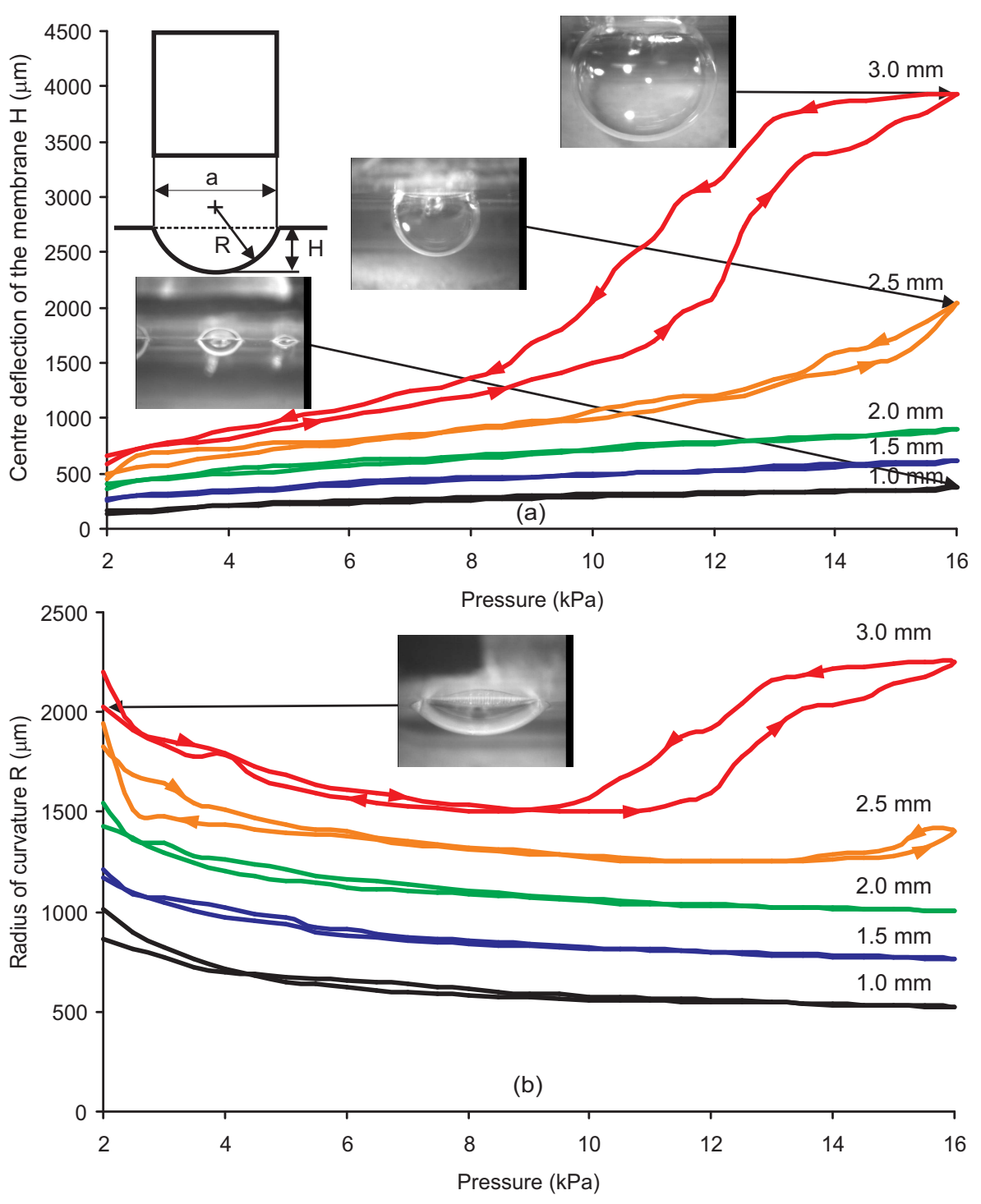

Figure 5: Geometry of square membranes at different applied pressures (the membrane size is indicated next to the respective curve): (a) Centre deflection; (b) Radius of curvature. 

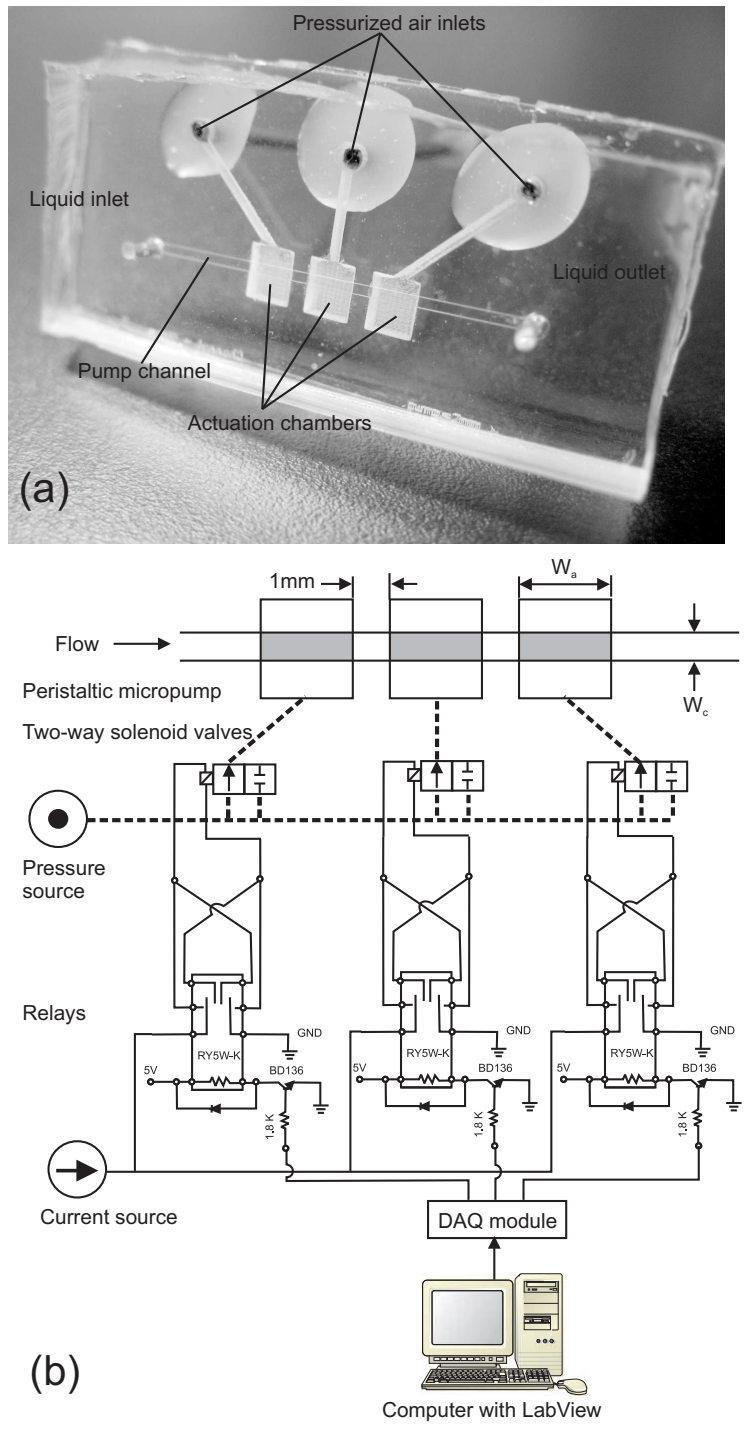

Figure 6: The PMMA/PDMS peristaltic micropump: (a) The assembled pump; (b) Control circuit and the experimental setup for characterizing the peristaltic micropump. The schematic of the micropump is shown on top. The active areas of the pump chambers are shaded grey. The size of the pump chamber is determined by the width of the pump channel $W_{\mathrm{c}}$ and the width of the actuation chamber $W_{\mathrm{a}}$. 

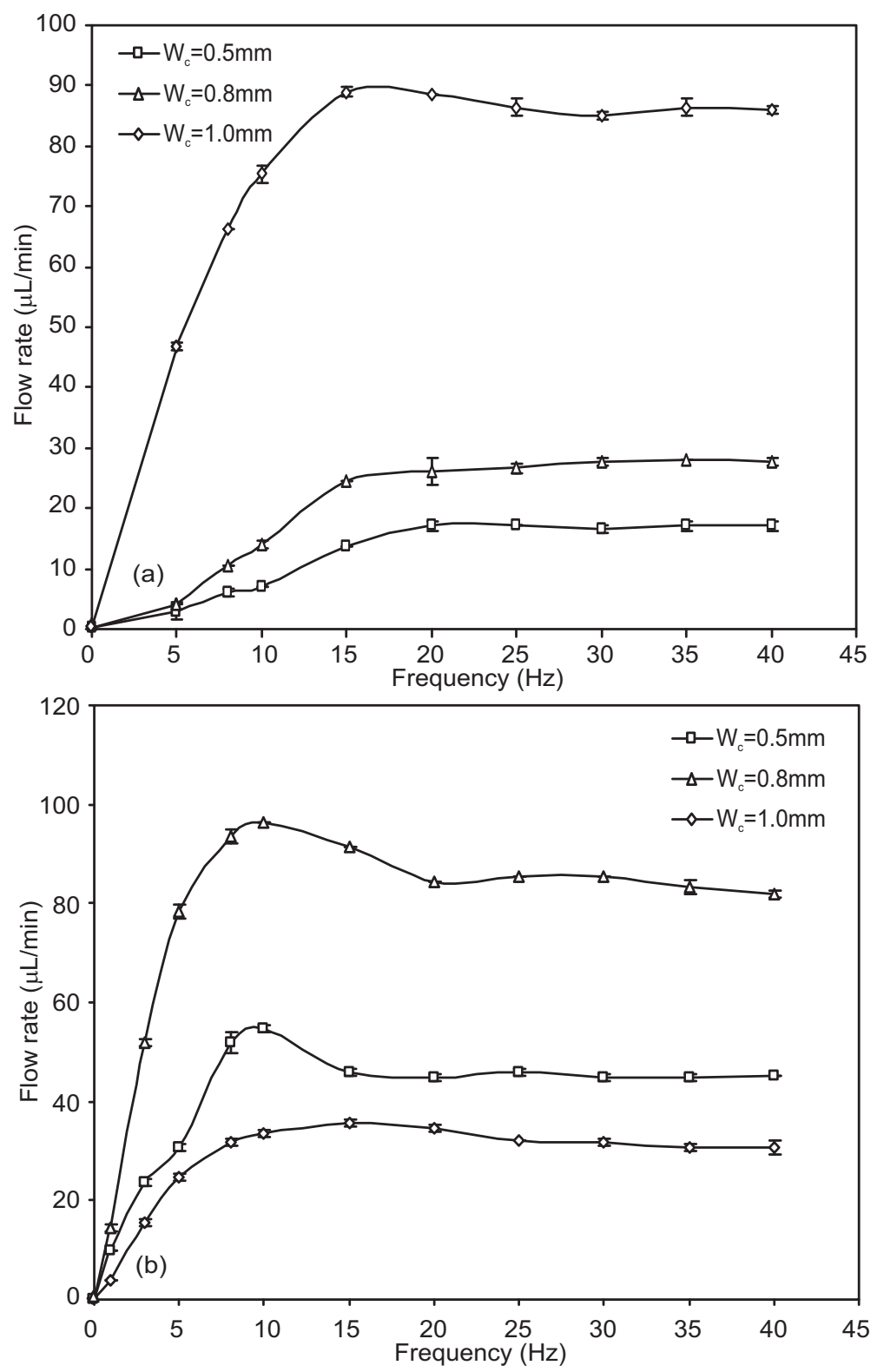

Figure 7: Performance of peristaltic micropumps with different channel widths $W_{\mathrm{c}}$ : (a) Pneumatic chamber with a width of $W_{\mathrm{a}}=1 \mathrm{~mm}$; (b) Pneumatic chamber with a width of $W_{\mathrm{a}}=2 \mathrm{~mm}$. 


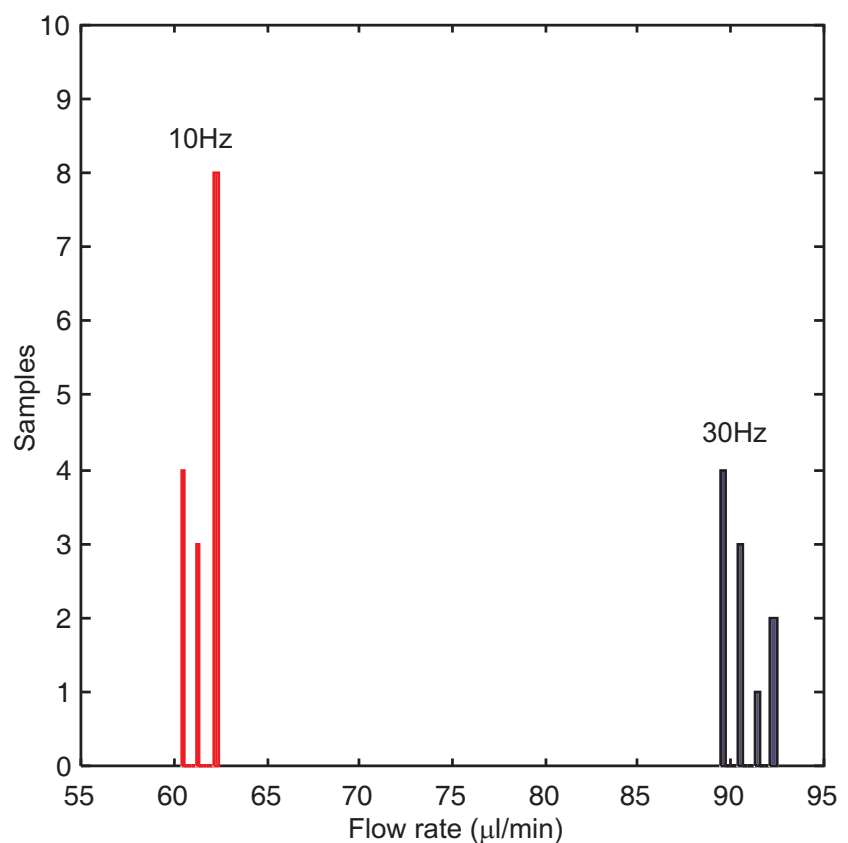

Figure 8: Flow rates at $10 \mathrm{~Hz}$ and $30 \mathrm{~Hz}$ sampled from pump operation over three days $\left(W_{\mathrm{c}}=0.5 \mathrm{~mm}\right.$ and $\left.W_{\mathrm{a}}=2 \mathrm{~mm}\right)$. 


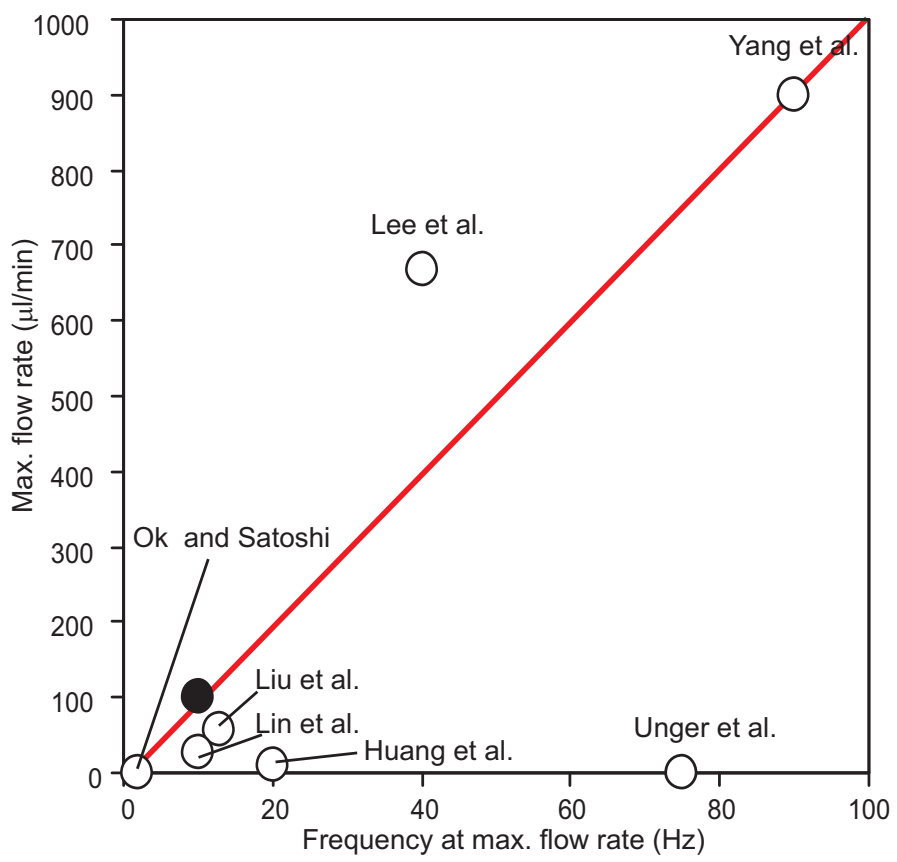

Figure 9: The performance of our micropump compared to other peristaltic micropumps reported in literature. 